\title{
Update on the treatment of narcolepsy: clinical efficacy of pitolisant
}

This article was published in the following Dove Press journal:

Nature and Science of Sleep

26 April 2017

Number of times this article has been viewed

\author{
Michael W Calik ${ }^{1,2}$ \\ 'Department of Biobehavioral Health \\ Science, ${ }^{2}$ Center for Narcolepsy, Sleep \\ and Health Research, University of \\ Illinois at Chicago, Chicago, IL, United \\ States
}

\begin{abstract}
Narcolepsy is a neurological disease that affects 1 in 2,000 individuals and is characterized by excessive daytime sleepiness (EDS). In 60-70\% of individuals with narcolepsy, it is also characterized by cataplexy or a sudden loss of muscle tone that is triggered by positive or negative emotions. Narcolepsy decreases the quality of life of the afflicted individuals. Currently used drugs treat EDS alone (modafinil/armodafinil, methylphenidate, and amphetamine), cataplexy alone ("off-label" use of antidepressants), or both EDS and cataplexy (sodium oxybate). These drugs have abuse, tolerability, and adherence issues. A greater diversity of drug options is needed to treat narcolepsy. The small molecule drug, pitolisant, acts as an inverse agonist/ antagonist at the $\mathrm{H}_{3}$ receptor, thus increasing histaminergic tone in the wake promoting system of the brain. Pitolisant has been studied in animal models of narcolepsy and used in clinical trials as a treatment for narcolepsy. A comprehensive search of online databases (eg, Medline, PubMed, EMBASE, the Cochrane Library Database, Ovid MEDLINE, Europe PubMed Central, EBSCOhost CINAHL, ProQuest Research Library, Google Scholar, and ClinicalTrials.gov) was performed. Nonrandomized and randomized studies were included. This review focuses on the outcomes of four clinical trials of pitolisant to treat narcolepsy. These four trials show that pitolisant is an effective drug to treat EDS and cataplexy in narcolepsy.
\end{abstract}

Keywords: narcolepsy, pitolisant, histamine

\section{Plain language summary}

Narcolepsy is a disease that affects 1 in 2,000 individuals in North America and Europe. These individuals suffer from excessive daytime sleepiness, and some also experience cataplexy or sudden loss of muscle control. These symptoms of narcolepsy cause a significant decrease in the quality of life for these narcoleptic individuals. There are limited drug options for narcoleptic individuals, and the drugs available have side effects that might be intolerable. Recent evidence suggests that activating neurons that release histamine in the brain can treat the symptoms of narcolepsy. The drug, pitolisant, has been shown to activate histaminergic neurons. This review focuses on the clinical outcomes of pitolisant, the newest drug approved for the treatment of the symptoms of narcolepsy.

\section{Introduction}

\section{Current state of narcolepsy treatment}

Narcolepsy, which affects $0.03-0.05 \%$ of individuals in North America and Europe, is a neurological disease characterized by excessive daytime sleepiness (EDS). ${ }^{1}$ Additionally, affecting $60-70 \%$ of narcoleptic individuals, ${ }^{2}$ narcolepsy can occur with cataplexy or a sudden loss of muscle tone that is triggered by positive or negative emotions.

Tel + I $3124|3058|$

Email mcalik@uic.edu 
Other signs of narcolepsy are hypnagogic hallucinations and sleep paralysis. ${ }^{1}$ Due to these symptoms, the quality of life of narcoleptic individuals is lower than that of the general population. ${ }^{3}$ Pathophysiologically, narcolepsy with cataplexy is associated with loss of orexin-producing neurons with low or undetectable orexin A levels in cerebrospinal fluid (CSF) and is labeled as type 1 narcolepsy. ${ }^{4}$ Narcolepsy without cataplexy, or type 2 narcolepsy, is associated with normal levels of orexin A in CSF, and this diagnosis is made only when type 1 narcolepsy is ruled out. ${ }^{1,4}$ Narcolepsy is also associated with low levels of CSF histamine, a wake-promoting amine. ${ }^{5}$ The prevalence and incidence of type 1 narcolepsy are between 25 and 50 per 100,000 people and 0.74 per 100,000 personyears, respectively. ${ }^{6}$ The prevalence and incidence of type 2 narcolepsy are between 42 and 73 per 100,000 people and 1.73 per 100,000 person-years, respectively. ${ }^{6}$ Women are at slightly higher risk of developing narcolepsy compared with men. ${ }^{6}$ Prevalence of narcolepsy does not differ in terms of race or ethnicity. ${ }^{7}$ Several etiological factors associated with narcolepsy include excessive alcohol use, obesity, associated diseases such as type 2 diabetes and cardiovascular diseases, timing of birth, and genetic/immune associations (eg, HLADQB1*0602; vaccines). ${ }^{6,8}$

Current pharmacotherapies focus on alleviating EDS or, when present, cataplexy, or both. Only one drug, sodium oxybate, is approved by the US Food and Drug Administration (FDA) to treat both EDS and cataplexy. Sodium oxybate is often well tolerated and does improve the quality of life of narcoleptic patients. However, sodium oxybate has abuse potential due to its anxiolytic, hypnotic and euphoric effects, ${ }^{9}$ and has possible neurotoxic side effects. ${ }^{1}$ Sodium oxybate, due to its short half-life, must be taken in a split dose, once at bedtime and again 2.5-4 hours later. Consequently, sodium oxybate treatment adherence is difficult. Other FDA-approved treatments for narcolepsy treat EDS only. The first-, second-, and third-line treatments for EDS in narcoleptic patients are modafinil/armodafinil, methylphenidate, and amphetamine, respectively. The first is preferred due to low abuse potential and lack of sympathomimetic side effects compared with the latter two, which are categorized as stimulants of the central nervous system. To treat cataplexy in narcoleptic individuals, antidepressants have been prescribed "off-label." However, there are no clinical trials on the efficacy or safety of antidepressants in the treatment of cataplexy. ${ }^{1}$ These drugs may also be combined to improve patient outcomes. ${ }^{10}$ Due to the scarcity of viable treatments for narcolepsy with or without cataplexy, there is a pressing need to develop new pharmacotherapies for this disease that affects 1 in 2,000 individuals.
Narcolepsy is the disruption of the brain wake-promoting system. ${ }^{11}$ The main cause of the disruption is the loss of wakepromoting orexinergic neurons in the lateral hypothalamus. ${ }^{1}$ These orexinergic neurons intermingle with the histaminergic neurons located in the tuberomammillary nucleus of the hypothalamus, which are also essential for the wakepromoting system of the brain. ${ }^{12}$ Current therapies, such as modafinil, stimulate the wake-promoting system of the brain by indirectly activating the histaminergic system by removing GABAergic inhibitory input on these neurons. ${ }^{11}$ Direct activation of orexin receptors on histaminergic neurons is a plausible therapy. However, no small molecule agonist for the orexin receptors has yet been discovered, and an exogenous peptide or prodrug precursor for the orexin receptors would not penetrate the blood-brain barrier (BBB). ${ }^{1}$ Another avenue of activating the brain wake-promoting system is by directly activating histaminergic neurons via antagonism of the $\mathrm{H}_{3}$ receptor, a histamine autoreceptor located presynaptically that controls histamine turnover by feedback inhibition of histamine synthesis and release. ${ }^{13}$ Antagonism of $\mathrm{H}_{3}$ receptor, through disinhibition of this autoreceptor, enhances synaptic histamine signaling on $\mathrm{H}_{1}$ receptors and promotes wakefulness. ${ }^{14}$ The first inverse agonist/antagonist of the $\mathrm{H}_{3}$ receptor to be tested in humans was pitolisant (BF2.649/tiprolisant/ Wakix $\left.^{\circledR}\right) .{ }^{15}$ Pitolisant was recently approved in the European Union for the treatment of both type 1 and type 2 narcolepsy. ${ }^{16}$

The objective of this review is to evaluate the latest results of the treatment of narcolepsy with pitolisant.

\section{Pharmacology of pitolisant}

In preclinical studies, pitolisant acted as a high-affinity competitive antagonist $\left(\mathrm{K}_{\mathrm{i}} 0.16 \mathrm{nM}\right)$ and as an inverse agonist $\left(\mathrm{EC}_{50} 1.5 \mathrm{nM}\right)$ at the human histamine $\mathrm{H}_{3}$ receptor subtype. Pitolisant showed high selectivity for $\mathrm{H}_{3}$ receptors compared with the other histamine receptor subtypes $\left(\mathrm{H}_{1}\right.$, $\mathrm{H}_{2}$, and $\mathrm{H}_{4}$ subtypes) and showed a lack of interaction with other human receptors or channels. In animal experiments, pitolisant increased histaminergic and noradrenergic neuron activity, increased wakefulness at the expense of slow wave and rapid eye movement sleep, had low abuse potential, and had no effect on mechanical or thermal pain thresholds. ${ }^{16}$

The recommended dose of pitolisant is between 4.5 and $36 \mathrm{mg}$ per day, depending on the patient's response. ${ }^{17} \mathrm{In}$ healthy human individuals, pitolisant reaches peak plasma concentration $\left(\mathrm{C}_{\max }\right) \sim 1-3$ hours after oral administration and has a plasma half-life of $10-12$ hours. ${ }^{16-18}$ Due to its long half-life, pitolisant should be taken in the morning so as to not disrupt sleep in the evening. Pitolisant reaches a steady state 
after 5-6 days of repeated administrations. It exhibits $>90 \%$ serum protein binding and is approximately equally distributed between red blood cells and plasma. Though the metabolization of pitolisant is incompletely characterized, the major inactive metabolites, 5-aminovaleric acid, glycine conjugate of the acid metabolite of O-dealkylated desaturated pitolisant, and glucuronide of a ketone metabolite of monohydroxy desaturated pitolisant, are formed by the cytochrome P450 (CYP) enzymes CYP3A4 and CYP2D6. Pitolisant also inhibits CYP2D6 ( $\left.\mathrm{IC}_{50} 2.6 \mu \mathrm{M}\right)$ and induces CYP3A4, CYP1A2, and CYP2B6. It is primarily excreted via urine ( $\sim 63 \%)$, with the rest of the excretion occurring through expired air $(25 \%)$ and feces $(<3 \%) .{ }^{16,17}$ Dosage adjustments should be made in individuals with renal or hepatic impairment. In large doses, pitolisant can prolong the QTc interval; hence, patients with cardiac disease or individuals taking other drugs that may increase QTc interval or increase blood concentrations of pitolisant should be carefully monitored. In animal studies, pitolisant or its metabolites crossed the placenta and were excreted in breast milk; therefore, it should not be used during pregnancy or during breastfeeding unless the potential benefit outweighs the potential risk for the fetus or baby. ${ }^{17}$

\section{Methods}

\section{Search strategy and selection of studies}

A comprehensive search of the following online databases was performed with the keyword phrase, "pitolisant and narcolepsy": Medline, PubMed, EMBASE, the Cochrane Library Database, Ovid MEDLINE, Europe PubMed Central, EBSCOhost CINAHL, ProQuest Research Library, Google Scholar, and ClinicalTrials.gov.

Due to the sparsity of clinical interventions to treat narcolepsy with pitolisant, all clinical interventions, including, nonrandomized, randomized, controlled, and/or uncontrolled studies, that were published in a peer-reviewed journal were included in this review. Four studies met these criteria. The search also came up with results from abstracts presented at international conferences; only abstracts that have not been published in a peer-reviewed journal since they were presented were included $(\mathrm{N}=2)$. The search also came up with ongoing, or completed but not yet published, clinical trials $(\mathrm{N}=5)$, and those were referenced briefly.

\section{Results}

\section{Clinical efficacy of pitolisant}

There are four published and peer-reviewed reports on the clinical efficacy of pitolisant to treat narcolepsy. ${ }^{19-22}$ These reports include a pilot, prospective, comparative, sequential placebo controlled, single-blind, multicenter study, ${ }^{19}$ a double-blind, randomized, and placebo-controlled trial;20 a double-blind, randomized, and placebo-controlled study on the safety and efficacy of pitolisant to decrease cataplexy; ${ }^{21}$ and a report on off-label use in teenagers. ${ }^{22}$

In the pilot study, ${ }^{19}$ placebo treatment followed by 7 days of $40 \mathrm{mg}$ dose of pitolisant was used in 21 subjects. Sleepiness was assessed using the Epworth Sleepiness Scale (ESS), and a sleep diary was used to quantify diurnal sleep episodes/ duration, nocturnal sleep duration, and wake after sleep onset (WASO) number/duration. Compared to baseline (before start of drug administration) and to placebo, pitolisant significantly decreases ESS scores by $>4$ points. However, those treated with pitolisant still had an average ESS score of $>11$, suggesting that these individuals were still moderately sleepy. The authors of this study did note that 9 of the 21 patients "normalized" their ESS scores below 11 (ESS responders). Individuals taking pitolisant decreased the number of diurnal sleep episodes as well as duration when compared to placebo. The effect of decreased diurnal sleep episodes and duration was greatest at the end of the 1 week trial, suggesting a delay in reaching maximal efficacy of pitolisant. ${ }^{19}$ This corresponds with the pharmacological characteristic of this drug to reach a steady state after 5-6 days of repeated administrations. ${ }^{17} \mathrm{~A}$ concern of pitolisant is that it can disrupt nocturnal sleep due to its wake-promoting properties. Here, the authors show no differences in nocturnal sleep duration, WASO number, or duration compared to placebo, which is attributed to pitolisant being taken in the morning. The authors acknowledged that the preceding data were obtained from the subjective measure of a sleep diary. Since the participants of this pilot study were allowed to continue taking anticataplectic medication, no assessment on pitolisant's anticataplectic effect was made. Adverse events reported in this study included 11 patients experiencing 23 adverse events during the pitolisant treatment and 7 patients experiencing 13 adverse events during placebo treatment. The majority of adverse events occurred in the first 3 days of treatment and included headache, nausea, insomnia, malaise, hallucination, and sweating. None of the adverse events ceased the treatment protocol; however, one patient had a decrease in dosage of pitolisant. ${ }^{19}$ A limitation of this study was that polysomnographies (PSGs) were not completed to quantify changes, if any, in sleep architecture, sleep duration, or sleep efficiency.

This pilot study paved the way for a larger more objective study conducted by the same group of authors. ${ }^{20}$ This study was a randomized controlled trial (RCT) that included 94 narcoleptic participants divided into placebo $(\mathrm{N}=30)$, 
pitolisant $(\mathrm{N}=31)$, and modafinil $(\mathrm{N}=33)$ treatment groups (these individuals ceased their EDS drug treatments 14 days before the start of the trial). The modafinil treatment group was included to test the noninferiority of pitolisant to modafinil, a standard treatment for EDS in narcolepsy. This study lasted for 8 weeks with the first 3 weeks using a flexible dosing regimen (pitolisant: 10, 20, or $40 \mathrm{mg}$ and modafinil: 100,200 , or $400 \mathrm{mg}$ ), followed by 5 weeks of stable dosing. During the flexible dosing weeks, individuals started at the lowest dose of the drug. In the second and third weeks, with individual clinical efficacy and safety assessed by the authors, the dosage would be increased if tolerable. ESS was used to score sleepiness. Maintenance of wakefulness test (MWT), a polysomnographic procedure designed to measure the ability to stay awake in boring conditions, ${ }^{23,24}$ sustained attention to response task (SART), a procedure designed to measure the quality of the awake state via the ability to sustain attention, ${ }^{24}$ and sleep diaries to assess symptoms of cataplexy were also used. Sixty-one percent of the individuals were considered cataplectic during the trial. Thirty-five percent of the individuals were taking anticataplectic drugs before the trial and remained on those drugs during this trial. Pitolisant and modafinil had improvements in ESS scores compared to placebo, and pitolisant was not noninferior compared to modafinil. The rate of ESS score decrease during the 8-week trial was similar in the pitolisant and modafinil groups. Moreover, there was a higher percentage of narcoleptic individuals taking pitolisant who normalized their ESS scores (ESS score $\leq 10$ ) compared to placebo; pitolisant was not different compared to modafinil to normalize ESS scores. MWT scores also showed significant improvement in the pitolisant and modafinil groups compared to placebo; there was no difference between the pitolisant and modafinil treatment groups. Compared with placebo, the error scores for SART significantly improved with pitolisant and modafinil with no differences between pitolisant and modafinil. Daily rate of cataplexy, as derived from sleep diaries, significantly improved compared to placebo. Modafinil failed to decrease cataplectic symptoms compared to placebo; however, there was no difference in cataplectic rates between modafinil and pitolisant. This suggests that pitolisant might be superior to modafinil in the treatment of cataplectic symptoms. All treatment groups reported adverse events. For all three groups, headache was reported. For pitolisant, insomnia, abdominal discomfort, and nausea, while for modafinil, abdominal discomfort and nausea, diarrhea, dizziness, anxiety, and irritability were reported. Once again, a limitation of this study was that PSGs were not completed to objectively quantify any changes in sleep architecture, sleep duration, or sleep efficiency.

This same group published a more recent randomized, double-blind, and placebo-controlled study on the safety and efficacy of pitolisant to decrease cataplexy in 105 narcoleptic individuals, with 54 individuals assigned to the pitolisant treatment group and 51 individuals assigned to the placebo group. ${ }^{21}$ The treatment period lasted for 7 weeks, with the first 3 weeks of flexible dosing to increase or decrease pitolisant $(5,10,20$, or $40 \mathrm{mg})$ or placebo concentration, followed by 4 weeks of stable dosing. During the treatment period, five patients in the pitolisant group and nine patients in the placebo group discontinued treatment due to protocol deviation, lack of efficacy, lack of efficacy and adverse event, or for no reason given. Forty-nine patients taking pitolisant and 42 patients taking placebo completed the study. The primary end point was the change in average number of cataplexy attacks per week at the end of the treatment period compared to baseline ( 2 weeks before starting the treatment) as recorded in individual diaries. Cataplexy attacks were defined as sudden and transient episodes of partial or generalized loss of muscle tone. Secondary end points were ESS and MWT scores and number of hallucinations recorded in individual diaries. Patients taking pitolisant decreased their weekly cataplexy rate by $75 \%$ from baseline levels, while the placebo group decreased their rate by $38 \%$ from baseline levels. The difference between the pitolisant and placebo change in weekly cataplexy was significant. This study included 12 individuals $(\mathrm{N}=4$ in the pitolisant treatment group; $\mathrm{N}=8$ in the placebo group) who kept taking other anticataplectic medication (eg, sodium oxybate or antidepressants) during the drug trial, and the effect of pitolisant was unchanged. ${ }^{21}$ Hallucinations, a symptom of narcolepsy, ${ }^{1}$ also decreased with pitolisant treatment. ${ }^{21}$ Similar to previous literature, ${ }^{20}$ pitolisant had a greater improvement in ESS and MWT scores from baseline than placebo, and more of the patients on pitolisant decreased their ESS score $\leq 10$ (ESS responders). ${ }^{21}$ The number of patients reporting adverse events did not differ between the groups, ${ }^{21}$ and reported adverse events were similar in previous reports. ${ }^{20}$ There was no difference in adverse event intensity and recovery between the pitolisant and placebo groups. However, double the number of adverse events was considered treatment-related and was considered mild-to-moderate intensity. Depression, as quantified by the Beck Depression Inventory, decreased from baseline in the pitolisant treated group, but not in the placebo group. Selfreported duration of night awakenings did not differ among the groups, and there were no changes in hematological or 
cardiovascular parameters in either group. Though clinical efficacy of pitolisant on cataplexy was demonstrated in this study, the study authors note a high placebo effect on cataplexy rate, which the authors attribute to the well-known role of subjective and emotional factors in triggering cataplexy attacks. ${ }^{21}$ Similar to literature mentioned earlier, measurements of sleep architecture via PSG were not completed.

A recent meta-analysis of clinical trials of treatments for narcolepsy was presented as an abstract at the International Society for Pharmacoeconomics and Outcomes Research in 2016. The meta-analysis pooled data from nine RCTs with 1,398 adult patients randomized into six narcolepsy treatments, including placebo. Pitolisant showed significant reduction in sleepiness score compared to placebo. However, the meta-analysis also showed that modafinil or sodium oxybate, which would benefit from larger clinical trials, was more effective in prolonging wakefulness on MWT at 8 weeks. ${ }^{25}$ Larger RCTs for pitolisant need to be conducted to show noninferiority to standard treatments of narcolepsy.

Another study used pitolisant off-label to treat narcolepsy in four teenagers who were refractory to available treatments, including modafinil, methylphenidate, sodium oxybate, and amphetamine. Dosage of pitolisant was started at $10 \mathrm{mg}$, and dosage increased to $40 \mathrm{mg}$ if clinical efficacy was not achieved. One teenager remained on an anticataplectic drug. Measurements were obtained from an Adapted ESS for EDS, Cataplexy Severity Rating Score (CSRS) for severity of cataplexy, and MWT for the ability to stay awake. The adapted ESS scores significantly decreased $<10$ using pitolisant alone. However, authors decreased the adapted ESS scores further by combining pitolisant treatment with other EDS decreasing drugs in three of the teenagers. In three teenagers, the cataplectic attacks decreased in severity. A strength of this small study was that PSGs were conducted before and after pitolisant treatment, and there were no significant differences in sleep measurements. Minor adverse effects of pitolisant were observed only during the first week of treatment and included insomnia, headache, hot flashes, leg pain, and hallucinations. ${ }^{22}$

A summary of these trials is listed in Table 1.

\section{Results}

\section{Future clinical trials of pitolisant}

In 2016, two abstracts on pitolisant treatment of narcolepsy were presented at the 23rd Congress of the European Sleep Research Society. ${ }^{26,27}$ One abstract looked at the long-term (12 months) efficacy of pitolisant, with some individuals comedicating with other narcoleptic drugs, in an open-label trial. Again, ESS scores and cataplexy severity decreased. More notable was that $20.2 \%$ of the individuals dropped out of the trial due to lack of efficacy of pitolisant. ${ }^{26}$ In another open-label trial presented as an abstract at the same meeting, pitolisant decreased ESS scores. However, 39.3\% of the individuals ceased pitolisant treatment due to lack of efficacy. ${ }^{27}$ These abstracts reported no serious adverse events, and the reported adverse events were similar to peer-reviewed published investigations. No further comment can be made on these abstracts until they are peer-reviewed and published. A summary of these conference abstracts is listed in Table 1. According to the US National Institutes of Health's registry of clinical trials, there are five clinical trials recruiting, active, or completed for the treatment of narcoleptic symptoms of EDS and/or cataplexy using pitolisant alone or in combination with sodium oxybate or modafinil in adults or children. ${ }^{16}$

\section{Discussion \\ Pitolisant as a potential new drug treatment for narcolepsy}

Current published data on the treatment of narcolepsy with pitolisant, a small molecule acting as an antagonist/inverse agonist at the $\mathrm{H}_{3}$ receptor subtype, are promising. In this review, there are four peer-reviewed published trials, two conference abstracts, and five ongoing or unpublished clinical trials investigating the efficacy of pitolisant to treat EDS and/or cataplexy in narcolepsy. The three placebo-controlled experiments, all conducted by the same group of investigators, recruited adults from multiple sites in Europe with similar demographics and baseline characteristics, making the data very homogenous. This same group of investigators also presented the abstracts and conducted the ongoing clinical trials. Other investigators need to conduct high-quality clinical trials to replicate the findings not only in demographics and baseline characteristics that are similar to the reported studies but also in differing demographics and baseline characteristics. Some data already exist with the open-label trial of pitolisant to treat narcolepsy in teenagers rather than adults, and there are ongoing trials in narcoleptic children. Still, pitolisant shows significant improvements in ESS and MWT scores and a decrease in cataplexy severity and rate over placebo with few adverse events. However, adverse events are still present and may lead to discontinuation of pitolisant as a treatment. The question remains whether or not pitolisant is more efficacious with less adverse events than the current treatments available to treat narcolepsy. Only one drug, sodium oxybate, has been approved to treat both EDS and cataplexy, and it is very well tolerated. However, the 
Table I Pitolisant in the treatment of narcolepsy

\begin{tabular}{|c|c|c|c|c|c|}
\hline Trial & $\mathbf{N}$ & Treatment & Dose & Clinical efficacy & Adverse events \\
\hline \multicolumn{6}{|c|}{ Peer-reviewed publications } \\
\hline \multirow{3}{*}{$\begin{array}{l}\text { Single-blind, } \\
\text { sequential } \\
\text { placebo- } \\
\text { controlled }{ }^{19}\end{array}$} & & Placebo & I wk & & \\
\hline & 21 adults & & & & \\
\hline & & Pitolisant & $40 \mathrm{mg} \mathrm{I} \mathrm{wk}$ & $\begin{array}{l}\text { Decreased ESS scores and diurnal } \\
\text { sleep episodes and duration; no } \\
\text { change in nocturnal sleep duration } \\
\text { and WASO number/duration }\end{array}$ & $\begin{array}{l}\text { Headache, nausea, insomnia, } \\
\text { malaise, hallucination, } \\
\text { sweating }\end{array}$ \\
\hline \multirow{3}{*}{$\begin{array}{l}\text { Double-blind, } \\
\text { randomized, } \\
\text { parallel } \\
\text { placebo- } \\
\text { controlled }^{20}\end{array}$} & 30 adults & Placebo & $8 w k$ & & Headache \\
\hline & $3 I$ adults & Pitolisant & $\begin{array}{l}\text { Up to } 40 \mathrm{mg} \text { flexible } \\
\text { dosing for } 3 \mathrm{wk} \text {, stable } \\
\text { dosing for } 5 \mathrm{wks}\end{array}$ & $\begin{array}{l}\text { Decreased ESS and SART scores and } \\
\text { daily cataplexy rate; increased MWT } \\
\text { scores }\end{array}$ & $\begin{array}{l}\text { Headache, insomnia, } \\
\text { abdominal discomfort, nausea }\end{array}$ \\
\hline & 33 adults & Modafinil & $\begin{array}{l}\text { Up to } 400 \mathrm{mg} \text { flexible } \\
\text { dosing for } 3 \mathrm{wk} \text {, stable } \\
\text { dosing for } 5 \mathrm{wk}\end{array}$ & $\begin{array}{l}\text { Decreased ESS and SART scores, } \\
\text { and increased MWT scores, but not } \\
\text { noninferior to pitolisant }\end{array}$ & $\begin{array}{l}\text { Headache, abdominal } \\
\text { discomfort, nausea, diarrhea, } \\
\text { dizziness, anxiety, irritability }\end{array}$ \\
\hline \multirow{2}{*}{$\begin{array}{l}\text { Double-blind, } \\
\text { randomized, } \\
\text { parallel } \\
\text { placebo- } \\
\text { controlled }^{21}\end{array}$} & 54 adults & Placebo & $7 \mathrm{wk}$ & $\begin{array}{l}\text { Decreased weekly cataplexy rate } \\
\text { (placebo effect) }\end{array}$ & $\begin{array}{l}\text { Headache, somnolence, } \\
\text { irritability, apathy, dizziness }\end{array}$ \\
\hline & $5 I$ adults & Pitolisant & $\begin{array}{l}\text { Up to } 40 \mathrm{mg} \text { flexible } \\
\text { dosing for } 3 \mathrm{wk} \text {, stable } \\
\text { dosing for } 4 \mathrm{wk}\end{array}$ & $\begin{array}{l}\text { Decreased weekly cataplexy rate, } \\
\text { hallucinations, depression, and ESS } \\
\text { scores; increased MWT scores; } \\
\text { no change in duration of night } \\
\text { awakenings, and hematological or } \\
\text { cardiovascular parameters }\end{array}$ & $\begin{array}{l}\text { Headache, somnolence, } \\
\text { irritability, anxiety, nausea, } \\
\text { apathy, dizziness }\end{array}$ \\
\hline Open-label $^{22}$ & 4 teenagers & $\begin{array}{l}\text { Pitolisant alone, } \\
\text { or concomitant } \\
\text { use with other } \\
\text { narcoleptic drugs }\end{array}$ & $\begin{array}{l}\text { Up to } 40 \mathrm{mg} \text { to } \\
\text { achieve clinical } \\
\text { efficacy; 9-24 mo }\end{array}$ & $\begin{array}{l}\text { Decreased adapted ESS scores; no } \\
\text { changes in sleep measurements; } \\
\text { reduced frequency and intensity of } \\
\text { cataplexy in two teenagers }\end{array}$ & $\begin{array}{l}\text { Insomnia, headache, } \\
\text { hot flushes, leg pain, } \\
\text { hallucinations }\end{array}$ \\
\hline \multicolumn{6}{|c|}{ Conference abstracts } \\
\hline Open-label ${ }^{26}$ & 89 adults & $\begin{array}{l}\text { Pitolisant alone, } \\
\text { or concomitant } \\
\text { use with other } \\
\text { narcoleptic drugs }\end{array}$ & $\begin{array}{l}\text { Up to } 36 \mathrm{mg} \text { to } \\
\text { achieve clinical } \\
\text { efficacy; } 12 \mathrm{mo}\end{array}$ & $\begin{array}{l}2 \mathrm{I} \text { individuals dropped out due } \\
\text { to lack of efficacy; decreased ESS } \\
\text { scores; reduced partial and total } \\
\text { cataplexy attacks and hallucinations }\end{array}$ & $\begin{array}{l}\text { Headache, insomnia, anxiety, } \\
\text { weight gain, depression }\end{array}$ \\
\hline Open-label $^{27}$ & 61 adults & Pitolisant & & $\begin{array}{l}24 \text { individuals stopped treatment } \\
\text { due to lack of efficacy or side effects; } \\
\text { decreased subjective sleepiness and } \\
\text { ESS scores }\end{array}$ & $\begin{array}{l}\text { Insomnia, headache, lack of } \\
\text { appetite, nausea }\end{array}$ \\
\hline
\end{tabular}

Abbreviations: ESS, Epworth Sleepiness Scale; mo, months; MWT, maintenance of wakefulness; SART, sustained attention to response task; WASO, wake after sleep onset; wk, weeks

dosing regimen of sodium oxybate necessitates twice-nightly administration and can be difficult to adhere to for patients. Other drugs, like modafinil/armodafinil, methylphenidate, and amphetamine, treat only the EDS and have tolerability issues and/or abuse potential. Finally, antidepressants have been used "off-label" to treat cataplexy, but with no RCTs on their use, it is difficult to state if antidepressants are beneficial. These drugs have been combined to improve efficacy. Pitolisant has been shown to be equal in its efficacy to treat EDS compared to modafinil. There have been no studies published yet to show if pitolisant can be equal in its efficacy to decrease cataplexy compared to sodium oxybate. Larger studies, which are being completed, will further elucidate the potential of pitolisant as a treatment for narcolepsy. ${ }^{16}$ These studies will also clarify whether pitolisant in combination with other narcoleptic drugs can improve symptoms of narcolepsy. It is important to note that pitolisant is not the only inverse agonist/antagonist of the $\mathrm{H}_{3}$ receptor subtype currently being developed. ${ }^{28}$ Pitolisant and other future $\mathrm{H}_{3}$ receptor subtype inverse agonists/antagonists increase the treatment options that individuals with narcolepsy currently have. With a disease that decreases the quality of life for 1 in 2,000 individuals, the development of pitolisant as a treatment for narcolepsy is a step in the right direction in biomedicine. 


\section{Conclusion}

Clinical data from four trials using pitolisant to treat EDS and cataplexy in narcolepsy show promising results. These promising results of pitolisant increase the options that narcoleptic patients have to treat their disease. Currently, pitolisant is only approved in Europe to treat narcolepsy. Further studies are needed to gage the long-term efficacy of pitolisant to treat EDS and cataplexy in narcolepsy.

\section{Disclosure}

The author reports no conflicts of interest in this work.

\section{References}

1. Black SW, Yamanaka A, Kilduff TS. Challenges in the development of therapeutics for narcolepsy. Prog Neurobiol. Epub 2015 Dec 23.

2. Bassetti C, Aldrich MS. Narcolepsy. Neurol Clin. 1996;14(3):545-571.

3. Bogan R, Swick T, Mamelak M, et al. Evaluation of quality of life in patients with narcolepsy treated with sodium oxybate: use of the 36 -item short-form health survey in a clinical trial. Neurol Ther. 2016;5(2):203-213.

4. Swick TJ. Treatment paradigms for cataplexy in narcolepsy: past, present, and future. Nat Sci Sleep. 2015;7:159-169.

5. Nishino S, Sakurai E, Nevsimalova S, et al. Decreased CSF histamine in narcolepsy with and without low CSF hypocretin-1 in comparison to healthy controls. Sleep. 2009;32(2):175-180.

6. Longstreth WT Jr, Koepsell TD, Ton TG, Hendrickson AF, van Belle G. The epidemiology of narcolepsy. Sleep. 2007;30(1):13-26.

7. Maski K. Understanding racial differences in narcolepsy symptoms may improve diagnosis. Sleep. 2015;38(11):1663-1664.

8. Ahmed SS, Montomoli E, Pasini FL, Steinman L. The safety of adjuvanted vaccines revisited: vaccine-induced narcolepsy. Isr Med Assoc J. 2016;18(3-4):216-220.

9. Kamal RM, van Noorden MS, Franzek E, Dijkstra BA, Loonen AJ, De Jong CA. The neurobiological mechanisms of gamma-hydroxybutyrate dependence and withdrawal and their clinical relevance: a review. Neuropsychobiology. 2016;73(2):65-80.

10. Thorpy MJ, Dauvilliers Y. Clinical and practical considerations in the pharmacologic management of narcolepsy. Sleep Med. 2015;16(1): 9-18.

11. Sundvik M, Panula P. Interactions of the orexin/hypocretin neurons and the histaminergic system. Acta Physiol (Oxf). 2015;213(2): 321-333.

12. Haas HL, Sergeeva OA, Selbach O. Histamine in the nervous system. Physiol Rev. 2008;88(3):1183-1241.
13. De la Herran-Arita AK, Garcia-Garcia F. Current and emerging options for the drug treatment of narcolepsy. Drugs. 2013;73(16): 1771-1781.

14. Parmentier R, Anaclet C, Guhennec C, et al. The brain H3-receptor as a novel therapeutic target for vigilance and sleep-wake disorders. Biochem Pharmacol. 2007;73(8):1157-1171.

15. Schwartz JC, Lecomte JM. Clinical trials with H3-receptor inverse agonists: what they tell us about the role of histamine in the human brain. Neuropharmacology. 2016;106:35-36.

16. SyedYY. Pitolisant: first global approval. Drugs. 2016;76(13):1313-1318

17. European Medicines Agency. Wakix (Pitolisant): Summary of Product Charatcteristics. 2017. Available from: http://www.ema.europa. eu/docs/en_GB/document_library/EPAR_-_Product_Information/ human/002616/WC500204746.pdf. Accessed January 4, 2017.

18. Bialer M, Johannessen SI, Levy RH, Perucca E, Tomson T, White HS Progress report on new antiepileptic drugs: a summary of the Twelfth Eilat Conference (EILAT XII). Epilepsy Res. 2015;111:85-141.

19. Lin JS, Dauvilliers Y, Arnulf I, et al. An inverse agonist of the histamine $\mathrm{H}(3)$ receptor improves wakefulness in narcolepsy: studies in orexin-/mice and patients. Neurobiol Dis. 2008;30(1):74-83.

20. Dauvilliers Y, Bassetti C, Lammers GJ, et al. Pitolisant versus placebo or modafinil in patients with narcolepsy: a double-blind, randomised trial. Lancet Neurol. 2013;12(11):1068-1075.

21. Szakacs Z, Dauvilliers Y, Mikhaylov V, et al. Safety and efficacy of pitolisant on cataplexy in patients with narcolepsy: a randomised, double-blind, placebo-controlled trial. Lancet Neurol. 2017;16(3):200-207.

22. Inocente $\mathrm{C}$, Arnulf I, Bastuji $\mathrm{H}$, et al. Pitolisant, an inverse agonist of the histamine $\mathrm{H} 3$ receptor: an alternative stimulant for narcolepsycataplexy in teenagers with refractory sleepiness. Clin Neuropharmacol. 2012;35(2):55-60.

23. Mitler MM, Gujavarty KS, Browman CP. Maintenance of wakefulness test: a polysomnographic technique for evaluation treatment efficacy in patients with excessive somnolence. Electroencephalogr Clin $\mathrm{Neu}$ rophysiol. 1982;53(6):658-661.

24. van der Heide A, van Schie MK, Lammers GJ, et al. Comparing treatment effect measurements in narcolepsy: the sustained attention to response task, Epworth Sleepiness Scale and maintenance of wakefulness test. Sleep. 2015;38(7):1051-1058.

25. Taneja A, Saharia P, Chadha N, Rajput A, Goel A. A network metaanalysis on comparative efficacy and safety of investigational and approved therapies for the treatment of narcolepsy. Value Health. 2016;19(3):A60.

26. Dauvilliers Y, Arnulf I, Szakacs Z, et al. Long term use of pitolisant to treat narcolepsy: HARMONY III study. J Sleep Res. 2016;25:275.

27. Hidalgo H, Mayer G, Geisler P, Young P, Kallweit U. Pitolisant as therapeutic option for narcolepsy: results from the German compassionateprogram 2015/16. J Sleep Res. 2016;25:366.

28. Gemkow MJ, Davenport AJ, Harich S, Ellenbroek BA, Cesura A, Hallett $\mathrm{D}$. The histamine $\mathrm{H} 3$ receptor as a therapeutic drug target for CNS disorders. Drug Discov Today. 2009;14(9-10):509-515.
Nature and Science of Sleep

\section{Publish your work in this journal}

Nature and Science of Sleep is an international, peer-reviewed, open access journal covering all aspects of sleep science and sleep medicine, including the neurophysiology and functions of sleep, the genetics of sleep, sleep and society, biological rhythms, dreaming, sleep disorders and therapy, and strategies to optimize healthy sleep. The manuscript
Dovepress

management system is completely online and includes a very quick and fair peer-review system, which is all easy to use. Visit http://www. dovepress.com/testimonials.php to read real quotes from published authors. 